\title{
diffusion-îundarrentals
}

The Open-Access Journal for the Basic Principles of Diffusion Theory, Experiment and Application

\section{Driven Polymer Translocation through a Nanopore: a Manifestation of Anomalous Diffusion}

\author{
Johan Dubbeldam, Andrey Milchev, Vakhtang Rostiashvili, Thomas Vilgis
}

Max-Planck-Institut für Polymerforschung, Ackermannweg 10, 55128 Mainz, Germany, E-Mail: rostiash@.mpip-mainz.mpg.de

\section{Introduction}

Recently, single molecule experiment probing single-stranded DNA or RNA translocation through a membrane nanopore attracted widespread attention [1]. Nevertheless the physical nature of the translocation process is still not well understood.

In this report we suggest a unique physical picture based on the mapping of the $3 \mathrm{~d}-$ problem on a $1 \mathrm{~d}$ translocation coordinate $s$ (or a translocated number of segments). We study the translocation dynamics of a polymer chain threaded through a nanopore with and without an external force. By means of diverse methods (scaling arguments, fractional calculus and Monte Carlo) we show that the relevant dynamic variable, the translocation number of segments $s(t)$, displays an anomalous diffusion behaviour even in the presence of an external force. The anomalous dynamics of the translocation process is governed in both (i.e. with and without a force) cases by the same universal exponent $\alpha=2 /\left(2 v+2-\gamma_{1}\right)$, where $v$ is the Flory exponent and $\gamma_{1}$ - the surface exponent. The process is described by a fractional Fokker - Planck equation which is solved exactly in the interval $0<s<N$ ( where $N$ is a chain length) with appropriate boundary and initial conditions. We have obtained a closed analytic solution for the probability function $W(s, t)$ in terms of the polymer chain length $N$ and the applied drag force $f$. This solution also enables to derive the expression for the probability distribution of translocation times $Q(t)$ as well as the variation with time of statistical moments :

$<_{S}(t)>$ and $<_{S}^{2}(t)>-<_{S}(t)>^{2}$ which provide full description of the diffusion process. It is found that the translocation time in the absence of an external force goes as $\tau \propto N^{2 v+2-\gamma_{1}}$ whereas in the driven anomalous diffusion case $\tau \propto N^{2 v+1-\gamma_{1}} f^{-1}$. Also the corresponding time dependent statistical moments, $<_{s} \quad(t)>\propto t^{\alpha}$ and $<s^{2}(t)>\propto t^{2 \alpha}$, reveal unambiguously the anomalous nature of the translocation dynamics and permit direct measurement of $\alpha$ in experiments. These findings are tested

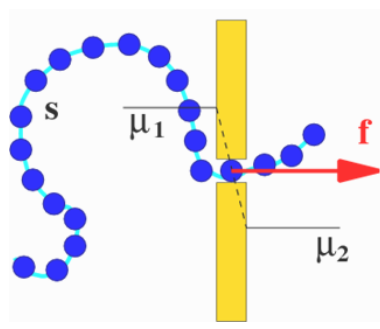

(a)

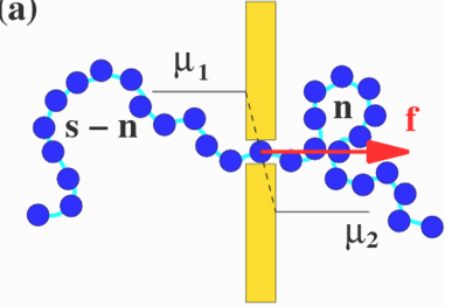
and found to be in perfect agreement with extensive Monte Carlo study.

Fig. 1: How a fold squeezes through a nanopore. The driving force $f$ is caused by a chemical potential gradient $\Delta \mu=\mu_{1}-\mu_{2}$.

(C) 2007, V. Rostiashvili

Diffusion Fundamentals 6 (2007) 14.1 - 14.2 


\section{Dynamics in terms of a translocation coordinate}

Figure 1 shows how a polymer fold of the length $s$ overcomes an entropic barrier $\Delta E(s)=\left(1-\gamma_{1}\right) T \ln s$ caused by a narrow pore.

This consideration enables to calculate the exponent of the anomalous diffusion $\alpha=2 v+2-\gamma_{1}$. For $d=3, v=0.588$ and $\gamma_{1}=0.680$ we obtain $\alpha=0.801$. Then for the free-force translocation time we have $\tau \propto N^{2 / \alpha} \approx N^{2.5}$. Remarkably, in $d=$ $2, v=0.75, \gamma_{1}=0.945$ and one finds $\alpha=0.783$, i.e. $\alpha$ is almost dimensionally independent! This explains why the measured exponents in $2 d$ [4] and in $3 d$ [5] are so close. The influence of external force $f$ could be treated within the formalism of fractional Fokker-Planck equation (FFPE) [6]. In our case the dimensionless force is caused by a chemical potential gradient $\Delta \mu=\mu_{1}-\mu_{2}$ (see Fig. 1). The analytical solution of FFPE for the translocation times distribution function $Q(t)$ has been compared with results of Monte Carlo study and Fig. 2 shows that the agreement between both methods is very good.

Fig. 2: First passage time distribution function at $N=128$ and different forces as calculated from $\mathrm{MC}$ - data (symbols) and the theoretical predictions (solid lines). The average translocation time which can be calculated from such curves (where the chain length ranges in the interval $32 \leq N \leq 256$ ) follows the law $\tau \propto f^{-1} N^{3 / 2}$.

\section{Conclusion}

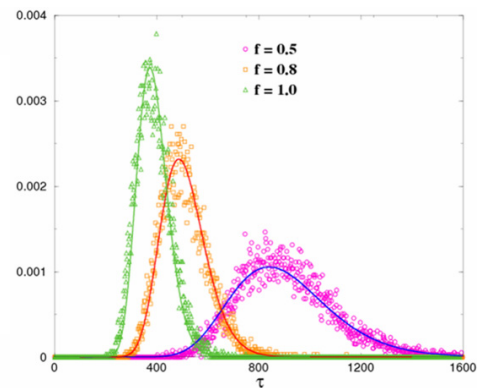

The polymer translocation is considered as squeezing of subsequent chain fragments (folds) through a narrow pore. This consideration gives rise to an universal scaling exponent for anomalous diffusion $\alpha=2 /\left(2 v+2-\gamma_{1}\right)$ so that the translocation time in the absence of drag scales as $\tau \propto N^{2 / \alpha}$. The presence of external force $f$ modifies this relationship to $\tau \propto f^{-1} N^{2 v+1-\gamma_{1}}$. This principal result is unambiguously confirmed by calculation of the mean first passage times (the average translocation times) from the derived analytic expression for translocation time distribution function $Q(t)$ as well as by comparison to the Monte Carlo simulation.

\section{References}

[1] A. Meller, J. Phys. Condensed Matter. 15 (2005) R581.

[2] J. Dubbeldam, A. Milchev, V. Rostiashvili, T. Vilgis, arXiv:cond-mat/0701664.

[3] J. Dubbeldam, A. Milchev, V. Rostiashvili, T. Vilgis, arXiv:cond-mat/0702463.

[4] J. Chuang, Y. Kantor, M. Kardar, Phys. Rev. E 65 (2004) 021806.

[5] D. Panija, G. Barkema, R.C. Ball, arXiv:cond-mat/0610671.

[6] R. Metzler, J. Klafter, Physics Rep. 339 (2000) 1. 